\title{
MicroRNA-331-3p inhibits proliferation and metastasis of ovarian cancer by targeting RCC2
}

\author{
Gulimire Buranjiang ${ }^{1}$, Reziya Kuerban², Ailikemu Abuduwanke², Xiaowen Li ${ }^{1}$, Gulina Kuerban ${ }^{1}$
}

\begin{abstract}
'Department of Gynecologic Oncology Radiation Therapy (Ward II), Xinjiang Medical University Third Clinical Medical College (Affiliated Tumor Hospital), Urumqi, Xinjiang, China

${ }^{2}$ Department of Gynecological Special Disease Clinic, Xinjiang Medical University Third Clinical Medical College (Affiliated Tumor Hospital), Urumqi, Xinjiang, China ${ }^{3}$ Department of Pediatric Ward, Xinjiang Uygur Autonomous Region People's Hospital, Urumqi, Xinjiang, China
\end{abstract}

Submitted: 27 June 2018

Accepted: 4 August 2018

Arch Med Sci 2019; 15 (6): 1520-1529

DOI: https://doi.org/10.5114/aoms.2018.77858

Copyright $\odot 2018$ Termedia \& Banach

\section{Abstract}

Introduction: Epithelial ovarian carcinoma (EOC) is one of the most lethal gynecologic malignancies, with a poor 5-year survival rate. Numerous studies have shown that microRNAs participate in the malignant behavior of ovarian cancer cells by directly targeting multiple oncogenes or tumor suppressor genes.

Material and methods: Reverse transcription-PCR was used to determine the level of miR-331-3p in EOC. Cells proliferation was measured with the Cell Counting Kit-8. Cell mobility were measured by wound-healing assay. Cell migration and invasion were measured by transwell assay. Luciferase assays were used to demonstrate that RCC2 was a directed target of miR-331-3p in EOC. Western blots were used to measure the protein expression.

Results: We found that the expression of microRNA-331-3p (miR-331-3p) in ovarian cancer cell lines is reduced $(p<0.01)$, and an increase of expression of miR-331-3p in ovarian cancer cells significantly inhibits cell proliferation $(p<0.001)$. Transwell and wound-healing assays showed that miR-331-3p inhibits the cell motility of ovarian cancer cells $(p<0.001)$. Regulator of chromosome condensation 2 (RCC2) was predicted to be a novel target for miR-331-3p. Our luciferase activity assay confirmed that RCC2 is directly targeted by miR-331-3p. RCC2 was negatively regulated by miR-331-3p $(p<0.001)$, and overexpression of RCC2 could restore the malignant behaviors of ovarian cancer cells, which was suppressed by miR-331-3p.

Conclusions: These data indicate that miR-331-3p can inhibit proliferation, migration, and invasion of ovarian cancer cells via directly targeting RCC2. Our study provides potential therapeutic targets for the treatment of ovarian cancer.

Key words: miR-331-3p, ovarian cancer, RCC2, cell proliferation, metastasis, migration.

\section{Introduction}

Ovarian cancer is one of the most common malignancies worldwide $[1,2]$. Each year approximately 140,000 people in the world die from ovarian cancer [3]. To date, staging/debulking surgery and taxane-plat-

\author{
Corresponding author: \\ Gulina Kuerban \\ Department of \\ Gynecologic Oncology \\ Radiation Therapy \\ (Ward II) \\ Xinjiang Medical \\ University \\ Third Clinical \\ Medical College \\ Affiliated Tumor Hospital \\ Urumqi, Xinjiang, China \\ E-mail: \\ GulinaKuerbanch01@163.com
}


inum-based chemotherapy are commonly used treatment modalities for ovarian cancer [4]. Despite advances in surgical techniques and chemotherapy, the 5 -year survival rate for ovarian cancer is still less than $50 \%[5,6]$. This poor prognosis is due to latent symptoms in the early stages, tumor resistance to chemotherapy, and a lack of effective early detection [7]. In order to improve the poor survival of ovarian cancer, a large number of studies are needed to unravel the molecular mechanism(s) responsible for the development of ovarian cancer.

MicroRNAs (miRNAs) are non-coding RNAs, composed of 20-23 nucleotides. miRNA binds to the $3^{\prime}$-UTR of a target gene and plays a negative regulatory role by inhibiting $\mathrm{mRNA}$ translation or inducing mRNA degradation $[8,9]$.

Accumulating studies indicated that miRNAs were involved in the progression of ovarian cancer and therefore could be used for cancer detection [10]. miRNAs have been reported to inhibit tumorigenesis of ovarian cancer by suppressing cell proliferation [11], migration [12], invasion [13, 14], and epithelial-to-mesenchymal transition [15]. In addition, miRNAs were also expected to be an important diagnostic tool $[16,17]$ as well as therapeutic targets for ovarian cancer $[18,19]$. Although a few studies have suggested that miR-331-3p can promote the growth of liver cancer [20, 21], miR-331-3p was taken as a suppressor in a variety of other human cancers. Studies showed that miR-331-3p could inhibit cervical cancer [22], gastric cancer [23], renal cell carcinoma [24], and colorectal cancer [25] by promoting apoptosis, suppressing cell proliferation, and inhibiting migration and invasion of tumor cells. However, the mechanism of miR-331-3p is still unclear in ovarian cancer.

The results of the current study showed that by inhibiting the target gene RCC2, miR-331-3p could significantly suppress tumor metastasis in an ovarian cancer cell line.

\section{Material and methods}

\section{Cell lines}

Of six human ovarian cancer cell lines, A2780 and SKOV3 were purchased from ATCC (Manassas, USA), and CAOV3, OVCAR3, ES-2, and COC1 were purchased from the Cell Center of Chinese Academy of Medical Sciences (Beijing, China). Two immortalized ovarian surface epithelial (IOSE) cells, IOSE80 and IOSE144, were purchased from BeNa Culture Collection (Suzhou, China). The 293T cell line was purchased from ATCC (Manassas, USA). Cells were cultured in appropriate growth media supplemented with $10 \%$ FBS and antibiotics at $37^{\circ} \mathrm{C}$ in presence of $5 \% \mathrm{CO}_{2}$ following the manufacturer's instructions.

\section{Reverse transcription-PCR (RT-PCR)}

The total RNA was extracted from cells using Trizol reagent (Invitrogen) according to the manufacturer's instructions. The primers for miR-331-3p and U6 were provided by the miRNA Real-Time PCR Assay kit (Genepharm, China). The primers for GAPDH and RCC2 were obtained from Sangon Biotech (Shanghai, China): GAPDH, forward, 5'-GCACCGTCAAGGCTGAGAAC-3', reverse, 5'-ATGGTGGTGAAGACGCCAGT-3'; RCC2 forward 5'-TTCCTTTGGGTGCCCTGAA-3', reverse 5'-GGCAGAATCTGTCCATCTTTCG-3'. The expression of RCC2 was detected by qRT-PCR (SYBR Green PCR kit, Bio-Rad). The relative expression level was determined by the $2^{-\Delta \Delta c t}$ method. U6 and GAPDH were used as controls. Each experiment was performed 3 times.

\section{Transfection of oligonucleotide}

Negative control (NC) mimics, miR-331-3p mimics, and miR-inhibitors were designed and synthesized by Genepharm (Shanghai, China). Cells were transfected with NC mimics, miR-331-3p mimics or miR-inhibitors using transfection reagent (pufei biotech, China) following the manufacturer's instructions.

\section{RCC2 overexpressing stable cells}

The RCC2 encoding sequence (without 3'-UTR) was cloned and inserted into the pcDNA3.1(+) vector (Invitrogen). Cell transduction was performed with Lipofectamine 2000 (Invitrogen) following the manufacturer's protocol, and RCC2 overexpressing stable cells were selected in the presence of $500 \mu \mathrm{g} / \mathrm{ml}$ of G418. The pcDNA3.1(+) vector was transfected into SKOV3 and A2780 cells and stable cell lines were selected as controls as described above.

\section{Cell proliferation}

Cell proliferation was measured with the Cell Counting Kit-8 (Dojindo, Kumamoto, Japan). The absorbance at $450 \mathrm{~nm}$ was measured at $0 \mathrm{~h}, 24 \mathrm{~h}$, $48 \mathrm{~h}$, and $72 \mathrm{~h}$; and then the growth curves were established to evaluate proliferation rates. Each experiment was performed 3 times.

\section{Wound-healing assay}

Cells were cultured in six-well plates and grown to $90 \%$ confluency. A $10 \mu$ pipette tip was used to make a wound on cell monolayers and then the cells were cultured in medium supplemented with $10 \%$ FBS. Wound healing was observed using a microscope (Leica, German) at $0 \mathrm{~h}, 24 \mathrm{~h}, 48 \mathrm{~h}$, and $72 \mathrm{~h}$. The wound healing rate was calculated using image pro plus 6.0 software. 


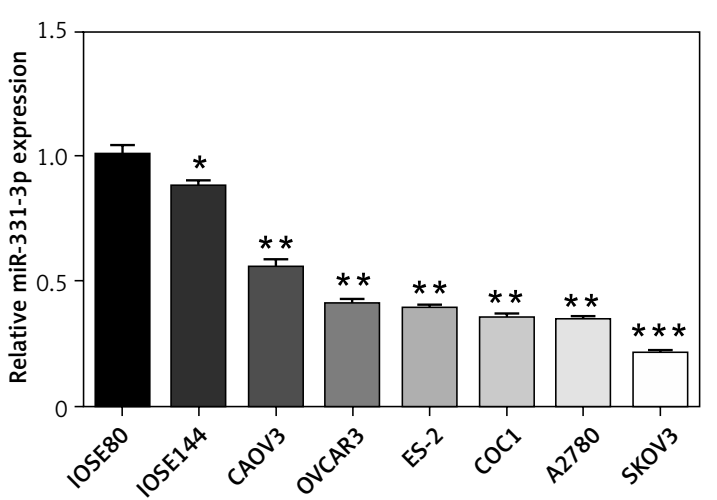

Figure 1. Expression of miR-331-3p in ovarian carcinoma. The relative expression levels of miR331-3p in six EOC cell lines (CAOV3, OVCAR3, ES-2, COC1, A2780, and SKOV3) and two immortalized ovarian surface epithelial cells (IOSE80, IOSE144) were determined by qRT-PCR. ${ }^{*} p<0.05,{ }^{* *} p<0.01$, ${ }^{* * *} p<0.001$

\section{Migration and invasion assay}

Cell migration and invasion were measured by transwell assay. Briefly, cells were trypsinized, washed and seeded on standard 24-well chambers (Corning Costar) at a seeding density of $1 \times 10^{5}$ cells per well, and FBS was added to the bottom chamber as a chemo-attractant. After $24 \mathrm{~h}$, chambers were fixed and stained with crystal violet, and the migrated cells were counted using a microscope. The invaded cells were counted after $48 \mathrm{~h}$. Data are shown as the mean value \pm SD from triplicate experiments.

\section{Luciferase assays}

A psiCHECK-2 vector (Promega) which contained the luciferase reporter gene and the 3'UTR of RCC2 (or its mutant sequence) was transfected into the 293T cell, and then miR-331-3p mimics or the control mimics were transfected into the same cells. After $48 \mathrm{~h}$, the cells were cracked and the firefly and Renilla fluorescence was detected by the Dual-Luciferase Reporter Assay System (Promega). Renilla fluorescence reflected the expression of 3'-UTR of RCC2. The experiments were performed independently in triplicate.

\section{Western blot}

The total protein was extracted from cells using NP-40 lysis buffer, supplemented with protease inhibitor cocktail (Roche Applied Science). A BCA protein quantitative kit (Beyotime) was used to detect protein concentration. The primary antibodies anti-RCC2 (Abcam) and anti-GAPDH (Abcam) were used in the western blot assays. All the experiments were performed in triplicate independently.

\section{Statistical analysis}

Data from three repeated experiments are presented as mean \pm SD. Student's $t$-test or ANOVA was conducted for statistical analysis. A $p$-value less than 0.05 was considered as statistically significant.

\section{Results}

\section{miR-331-3p is down-regulated in ovarian} cancer

To examine the expression of miR-331-3p in human ovarian cancer cells, total RNAs were isolated from six ovarian cancer cell lines (CAOV3, OVCAR3, ES-2, COC1, A2780 and SKOV3), and two immortalized ovarian surface epithelial cell lines (IOSE80 and IOSE144). Quantitative-PCR was performed to measure miR-331-3p expression. The expression levels of miR-331-3p were down-regulated in ovarian cancer cell lines, compared to that of the normal ovarian surface epithelial cells. Decreased miR-331-3p expression in ovarian cancer cell lines suggested miR-331-3p to be a tumor suppressor in ovarian cancer (Figure 1).

\section{miR-331-3p inhibits cell proliferation, migration and invasion}

In order to confirm the impact of miR-331-3p in ovarian cancer, miR-331-3p mimics were transfected into ovarian cancer cell lines A2780 and SKOV3. The expression of miR-331-3p was detected by RT-PCR. The results showed that miR-331-3p was upregulated in transfected cells (Figure $2 \mathrm{~A}$ ). Proliferation assays showed that miR-331-3p expression significantly inhibited cell proliferation in A2780 and SKOV3 cells (Figure 2 B). Transwell assays were utilized to examine the impact of miR-331-3p on EOC cell migration and invasion. The results showed that miR-331-3p mimics dramatically inhibited the migration abilities of SKOV 3 and A2780 cells (Figure 2 C). Similarly, the invasion abilities of SKOV 3 and A2780 cells were suppressed significantly after transfection with miR-331-3p mimics compared to that of cells transfected with NC mimics (Figure $2 \mathrm{D}$ ). Cell mobility in A2780 and SKOV3 was determined by wound-healing assays (Figure $2 \mathrm{E}$ ). The results showed that miR-331-3p mimics suppressed the cell mobility significantly in A2780 and SKOV3 cells. Images of transwell and wound healing assays are shown in Figure 3. These data suggested that miR-331-3p suppresses cell proliferation, migration and invasion of ovarian cancer.

\section{miR-331-3p directly targets RCC2}

In order to characterize the molecular mechanism by which miR-331-3p suppresses EOC growth, migration and invasion, we searched three target 
A
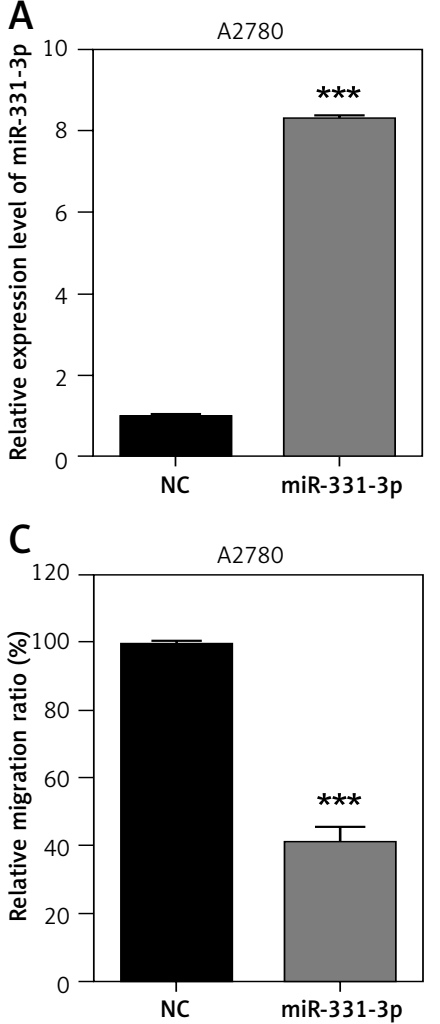

SKOV3
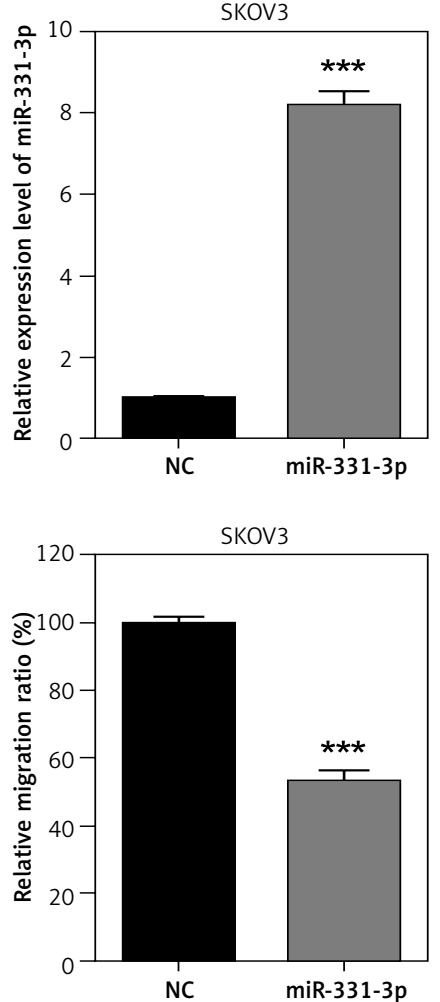

$\mathrm{A} 2780$

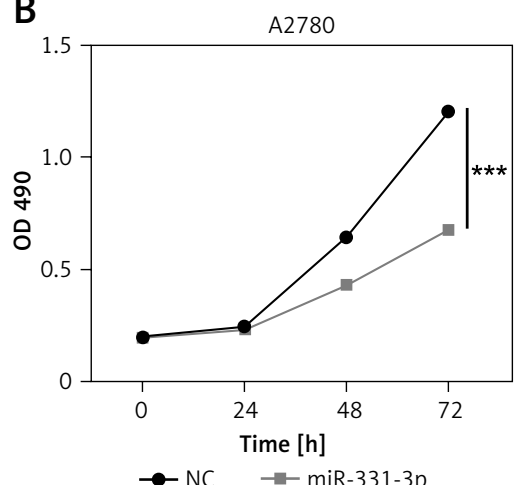

$\rightarrow \mathrm{NC} \quad \rightarrow-\mathrm{miR}-331-3 \mathrm{p}$

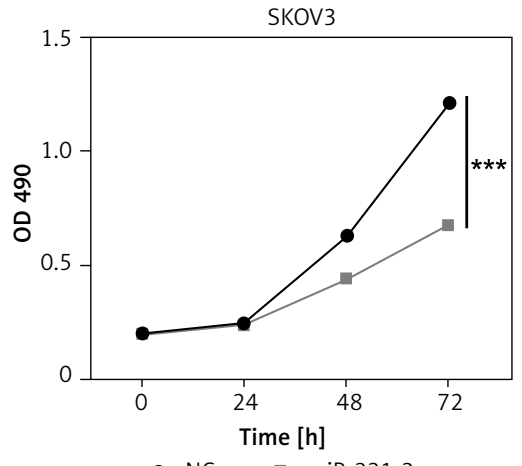

$\rightarrow$ NC $\rightarrow$ miR-331-3p

D
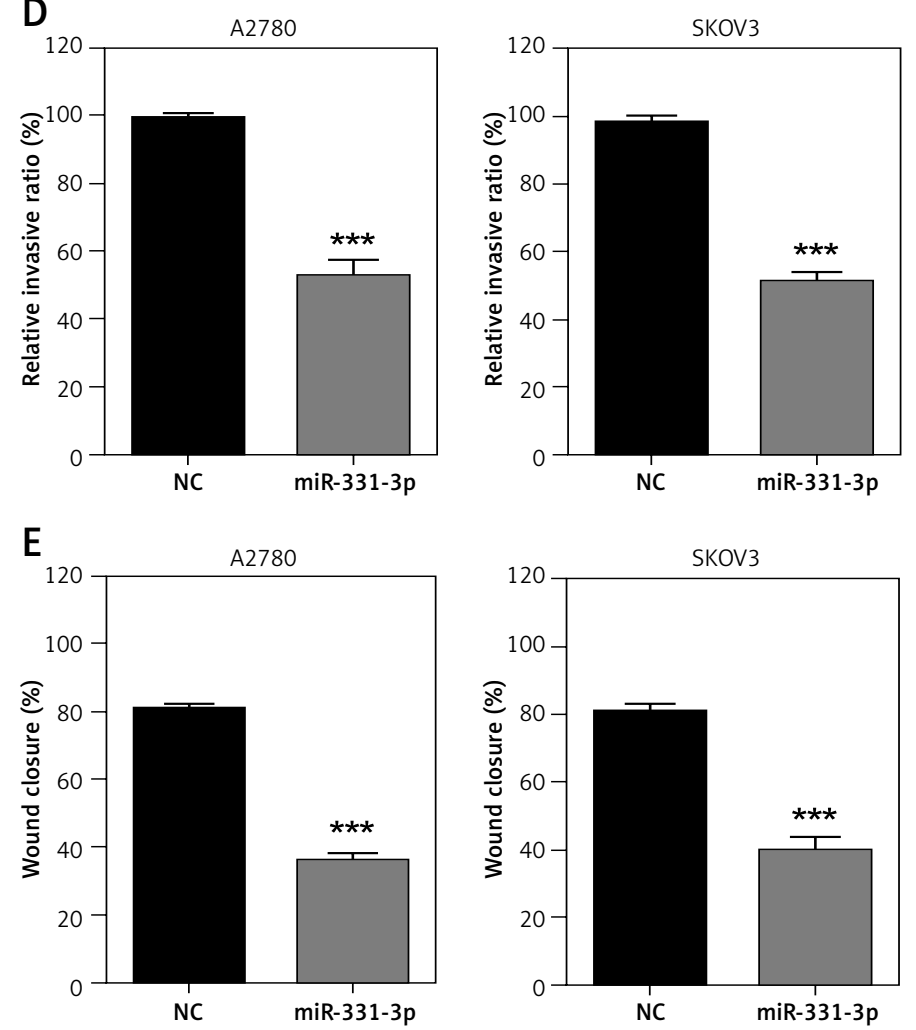

Figure 2. miR-331-3p suppresses EOC cell proliferation, migration and invasion. A- miR-331-3p level was detected by qRT-PCR. B - Proliferation of EOC cells treated with miR-331-3p or NC mimics was measured using Cell Counting Kit-8 (CCK-8). C - Transwell migration assay of EOC cells treated with miR-331-3p or NC mimics. D - Transwell invasion assay of EOC cells treated with miR-331-3p or NC mimics. E - Wound healing assays of EOC cells transfected with miR-331-3p or NC mimics 
A
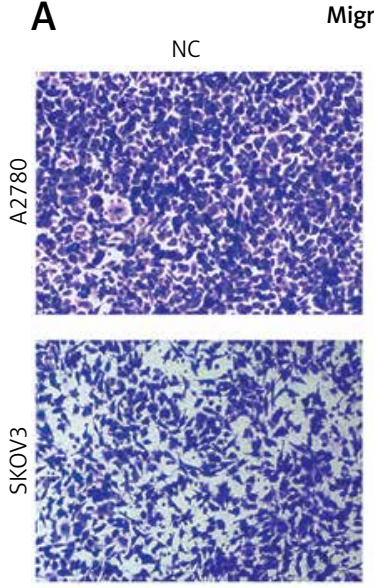

Migration
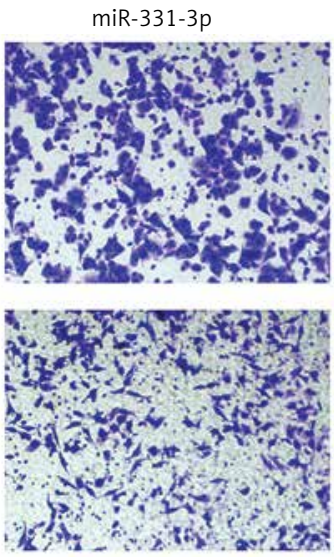

A2780
B
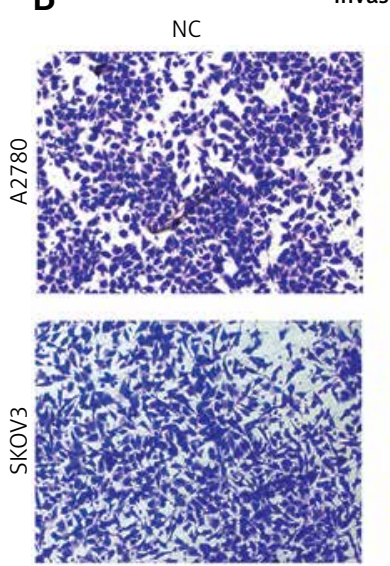

SKOV3

NC
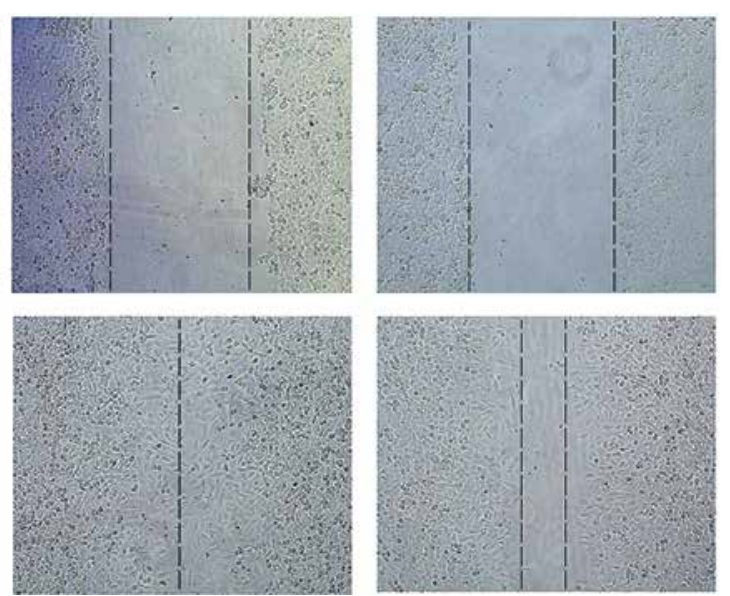

miR-331-3p
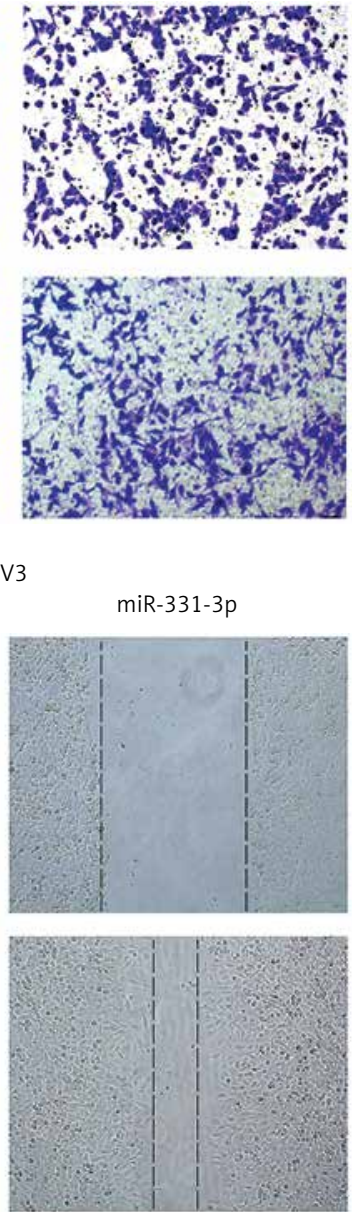

Figure 3. miR-331-3p inhibits migration and invasion of EOC cells. Images of transwell migration assay of EOC cells (A), transwell invasion assay of EOC cells (B), and wound healing assays of EOC cells transfected with miR-331-3p or NC mimics (C) are shown

scan databases - TargetScan, DIANA and miRanda - which predicted RCC2 as a direct target of miR331-3p (Figure 4 A). To confirm that miR-331-3p can target and suppress RCC2, a luciferase reporter vector containing the binding site of RCC2 3'-UTR, wild type (WT) or the mutant type (MUT) was constructed and transfected into 293T cells. The results of luciferase assays are shown in Figure $4 \mathrm{~B}$. In the WT group, miR-331-3p significantly suppressed the relative luciferase activities, whereas in the mutant type group, no significant difference in the relative luciferase activity was detected between NC and miR-331-3p treated cells. The results of the luciferase assay suggested that miR-331-3p suppresses RCC2 by identifying and binding specific sequences of RCC2 3'-UTR. The mRNA level (Figure 4 C) and protein level (Figure $4 \mathrm{D}$ ) of RCC2 in miR-331-3p transfected $\mathrm{A} 2780$ and SKOV3 were detected by RT-PCR and western blot respectively. The results showed that the expression of RCC2 was significantly inhibited by miR-331-3p at both the mRNA level and the protein level.
Restoration of RCC2 expression rescues the impact of miR-331-3p on EOC cells

To explore whether RCC2 is the target of miR331-3p in ovarian cancer; RCC2 was overexpressed in A2780 and SKOV 3 cells by transfection with recombinant vectors carrying RCC2 cDNA (without 3'-UTR) (Figure 5 A). Transwell and wound healing assays were conducted, and the results showed that the subsequent reintroduction of RCC2 without an intact 3'-UTR partly restored the inhibitory effects of miR-331-3p mimics on the cell proliferation, migration and invasion ability of A2780 and SKOV 3 cells (Figures 5 B-E, 6). Taken together, the data indicate that RCC2 acts as a functional target of miR-331-3p in EOC cells.

\section{Discussion}

Ovarian cancer is one of the world's most common gynecological malignant tumors [5, 26]. Despite recent advances in detection and cytotoxic therapies, more than $70 \%$ of ovarian cancer 
A

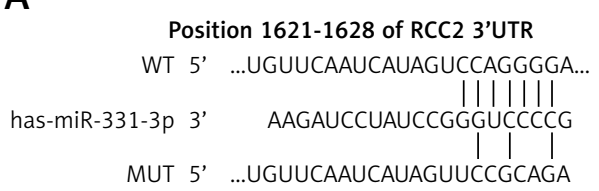

B

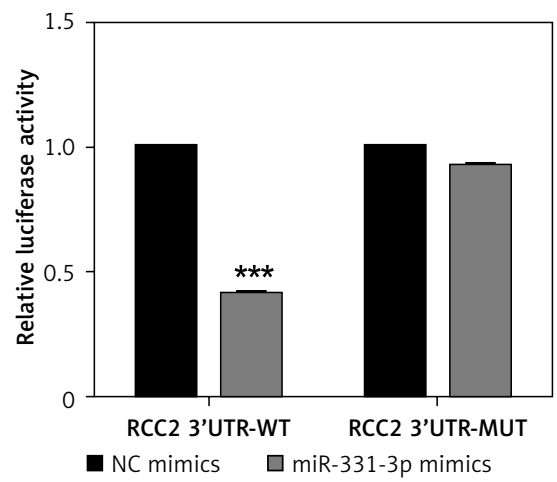

C

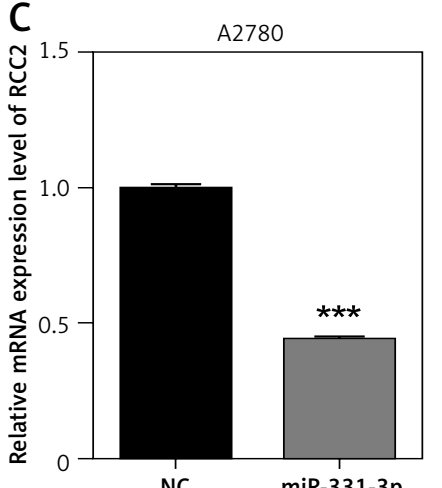

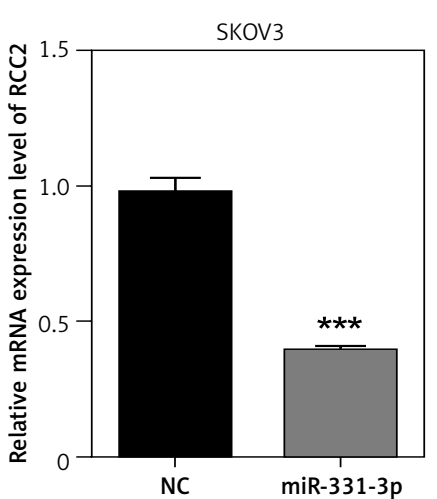

D

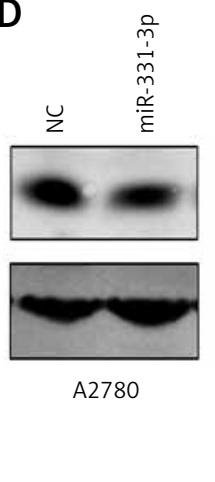

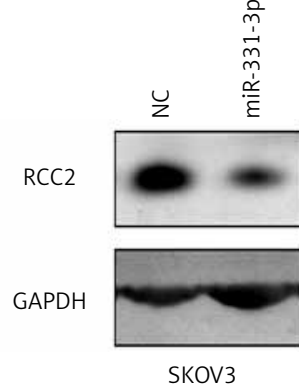

Figure 4. RCC2 is a direct target of miR-331-3p in EOC cells. A - Predicted binding sites of miR-331-3p in the 3'-UTR of RCC2. MUT (Mutant-type) was generated by mutating four nucleotides in the seed region of the $3^{\prime}$-UTR of RCC2. B - Luciferase report assays were performed in 293T cells, which were transfected with RCC2 3'-UTR WT or MUT vectors, and treated with miR-331-3p or NC mimics. C - The mRNA expression of RCC2 in A2780 and SKOV3 cells after $48 \mathrm{~h}$ transfection with miR-331-3p and NC mimics was measured by qRT-PCR. D - The protein level of RCC2 in A2780 and SKOV3 cells after $48 \mathrm{~h}$ transfection with miR331-3p and NC mimics was measured by western blot

cases were diagnosed at an advanced stage, and late diagnosis led to poor prognosis [27, 28]. Furthermore, patients with ovarian cancer are faced with a serious risk of recurrence, and the current standard treatment is unable to cure the disease $[6,29]$. Therefore, ovarian cancer still remains the leading cause of mortality in gynecologic cancer, which accounts for nearly 140 thousand deaths per year worldwide $[5,26]$. Thus, there is an urgent demand to develop new diagnostic and therapeutic targets for this devastating disease. In recent years, more and more miRNAs have been found to play an important role in the occurrence and development of ovarian cancer.

miR-331-3p was reported to suppress a wide variety of human cancers by inhibiting different target genes. In human renal carcinoma cells, miR-331-3p inhibits cell proliferation by targeting HER2 and NRP2 [24]; miR-331-3p has also been reported to inhibit cell proliferation, block the cell cycle and induce apoptosis in cervical cancer by regulating NRP2 [22], while in prostate cancer, miR-331-3p suppresses cell proliferation and migration by directly targeting RALA [30].

Our study found that miR-331-3p was significantly reduced in ovarian cancer cells compared to immortalized ovarian surface epithelial cell lines (Figure 1). These results suggested that miR-331-3p might act as a suppressor in ovarian cancer. In order to verify this hypothesis, miR-331-3p mimics were transfected into EOC cells (Figure $2 \mathrm{~A}$ ). CCK8, transwell, and wound healing assays were conducted, and the results indicated that miR331-3p suppressed EOC cell proliferation, migration, and invasion (Figures 2 B-E). In addition, regulator of chromosome condensation 2 (RCC2) was predicted to be a novel target of miR-331$3 p$. The luciferase assay verified that miR-331-3p negatively regulated $\mathrm{RCC} 2$ gene expression via specifically binding to the 3'-UTR (Figures 4 A, B). Results of RT-PCR and western blot suggested that the expression of RCC2 was downregulated by miR-331-3p in EOC cells (Figures 4 C, D). In addition, upregulated RCC2 was able to restore the cell proliferation, migration, and invasion in miR331-3p transfected cells (Figure 5). The results in Figure 5 suggested that RCC2 plays an important role in the miR-331-3p suppressing ovarian cancer. RCC2 was previously reported to be an important component of the chromosomal passenger complex (CPC); and CPC was regarded as an important regulator of chromosome and cyto- 

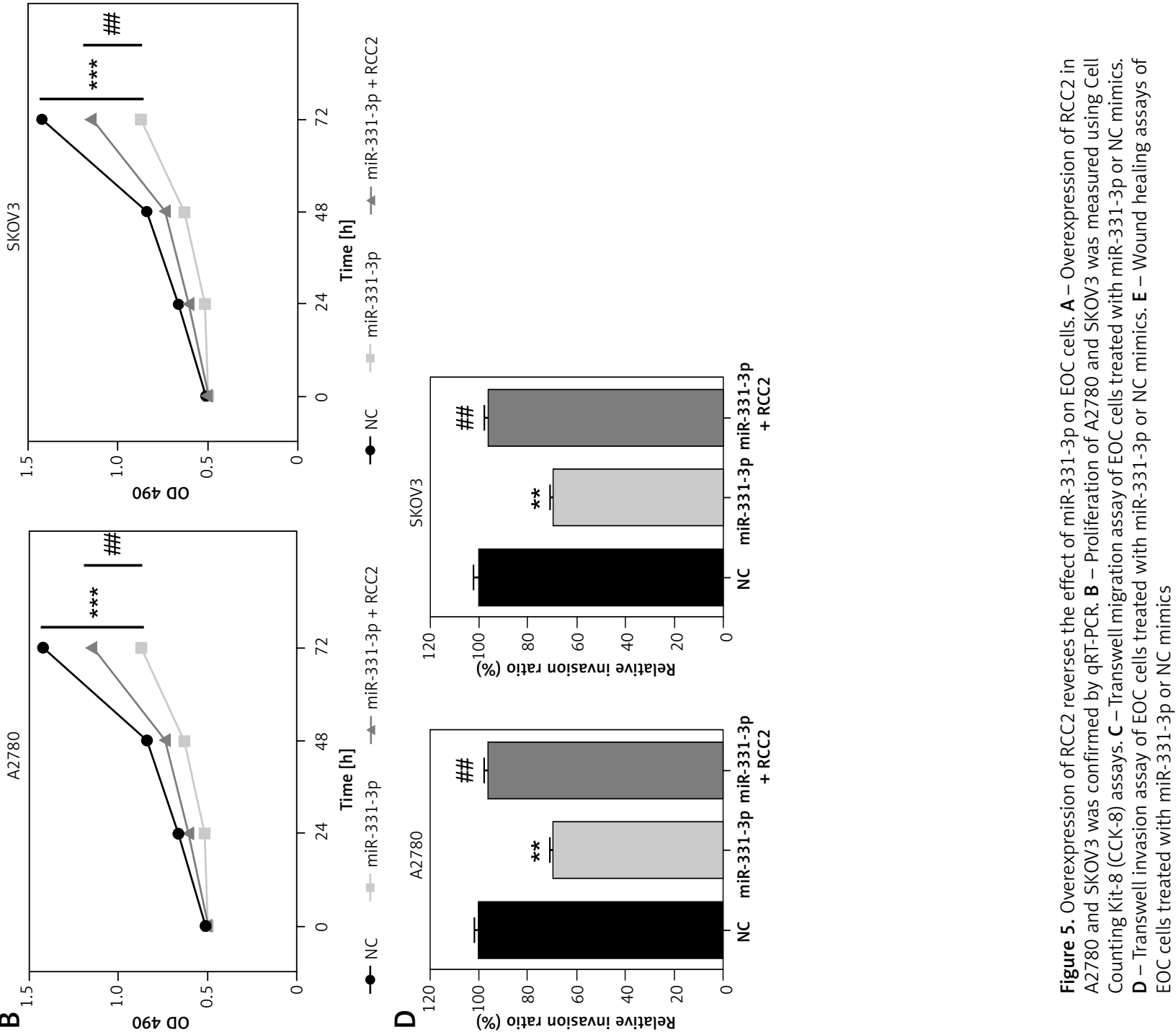

(\%) о!ฺеג ио!sели! әм!?ерәу
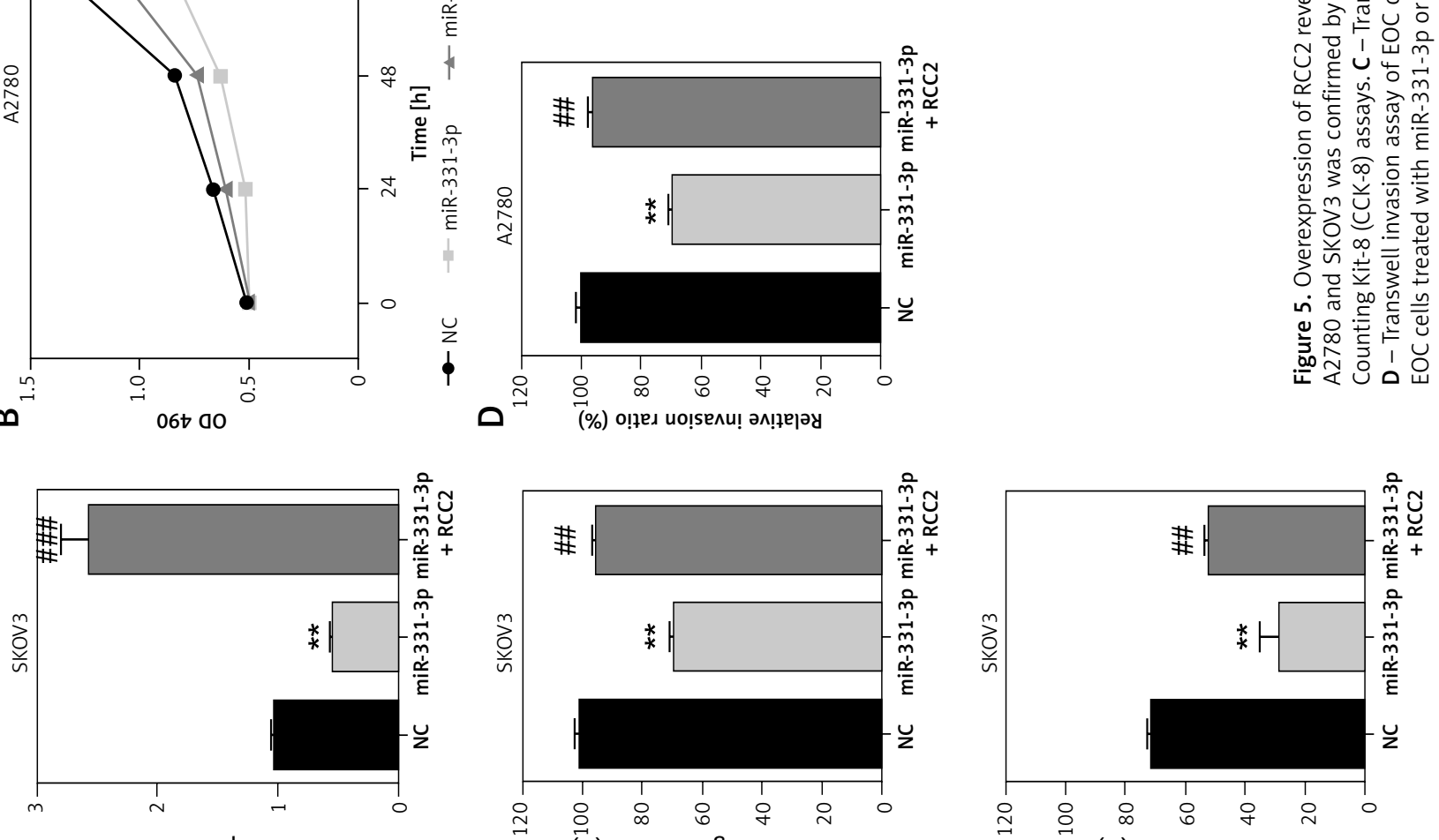

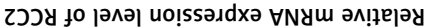

(\%) əunsolj punoM

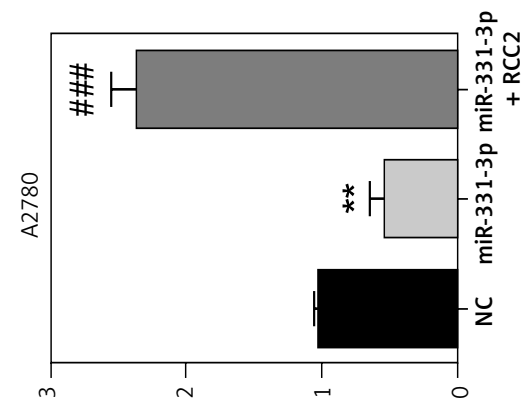

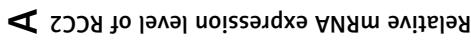
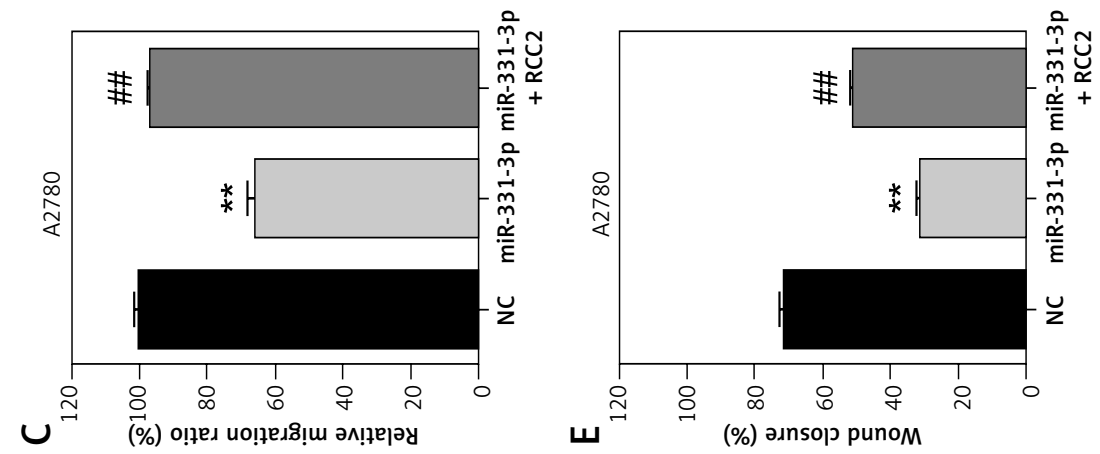
A
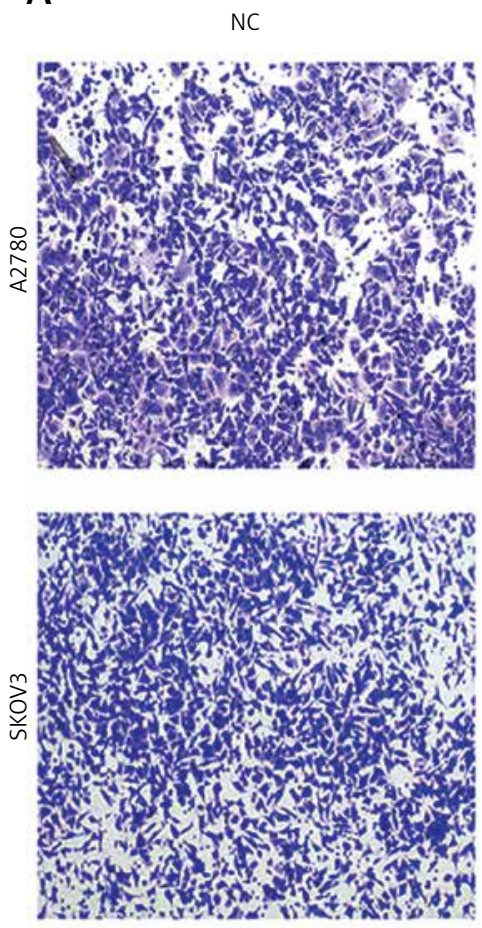

B

NC
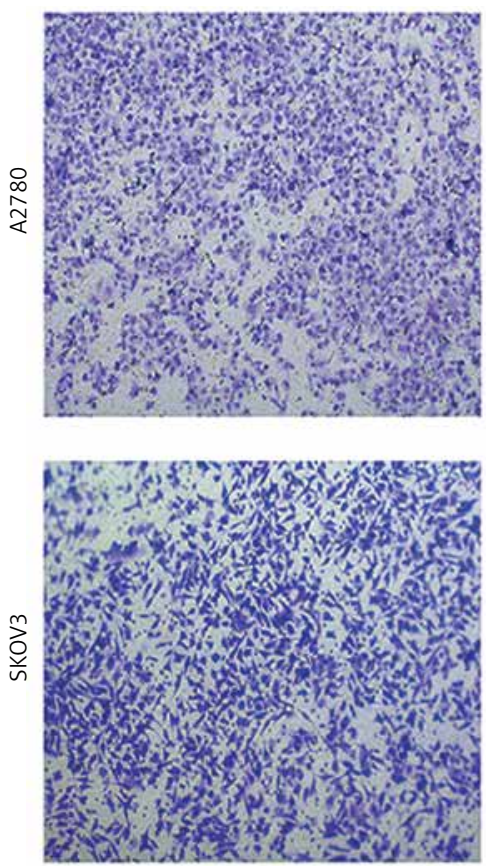

Migration

miR-331-3p
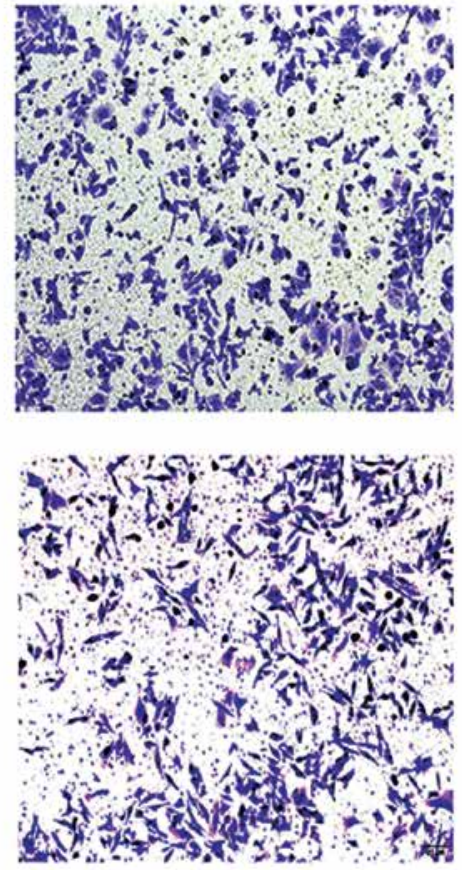

Invasion

miR-331-3p
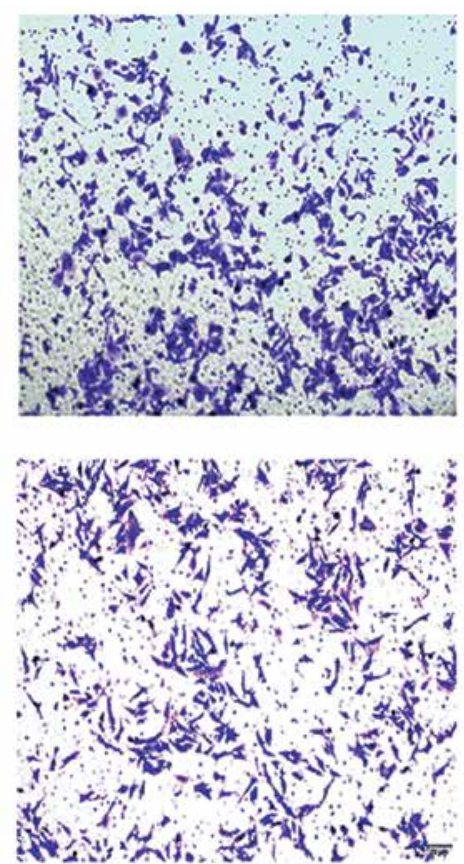

$\operatorname{miR}-331-3 p+R C C 2$
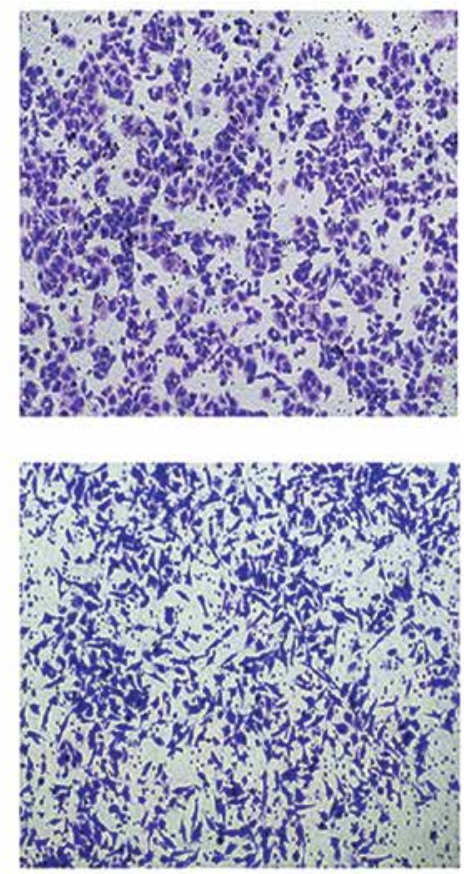

$\operatorname{miR}-331-3 p+R C C 2$
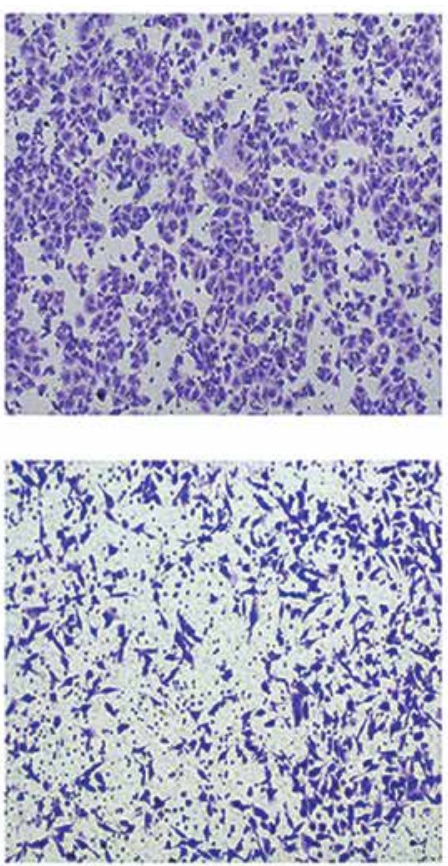

Figure 6. Overexpression of RCC2 restores the migration and invasion of EOC cells. Images of Figure 5 C (A), Figure $5 \mathrm{D}(\mathrm{B})$, and Figure $5 \mathrm{E}(\mathrm{C})$ are shown

skeleton formation as well as mitosis [31, 32]. In recent years, RCC2 has been reported as a tumor promoter in various cancers. RCC2 was shown to be upregulated in gastric carcinoma tissues, which can regulate the cell cycle process to promote the proliferation of cancer cells $[33,34]$. Another study reported that RCC2 knockdown arrested the cell cycle at prometaphase in HeLa cells, suggesting RCC2 to be a negative regulator of the cell cycle which was found to be driven by regulating chromosomal separation and cell division [35-37]. The molecular mechanism of RCC2 in ovarian cancer will be further verified in our future work. 
C A2780

$48 \mathrm{~h}$
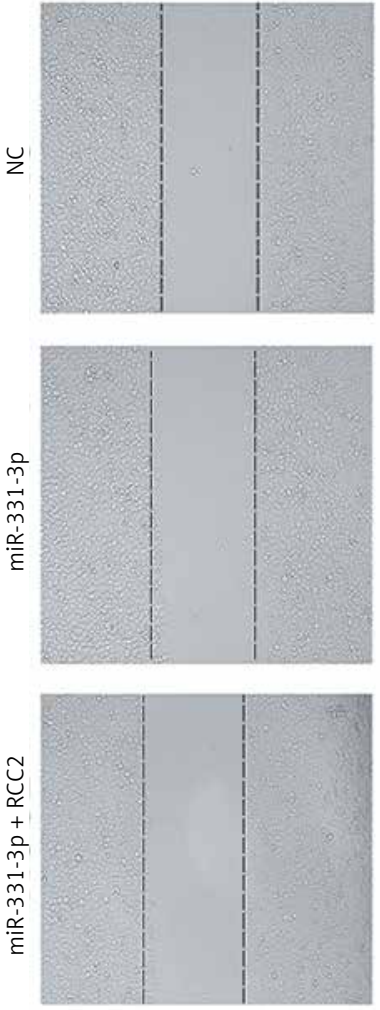
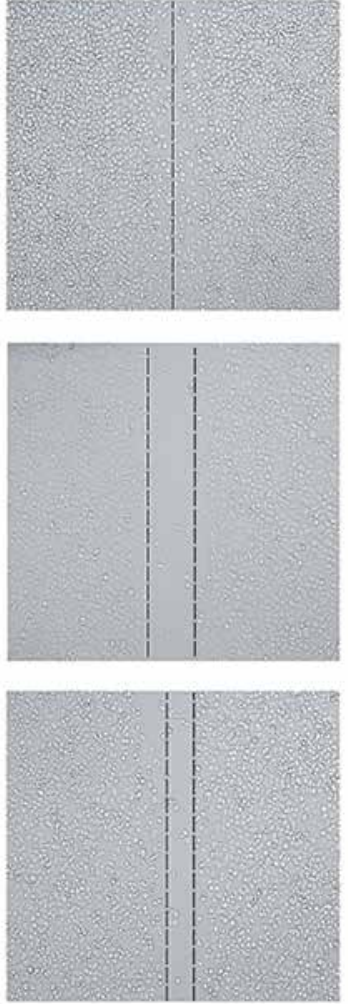

SKOV3

$\mathrm{Oh}$
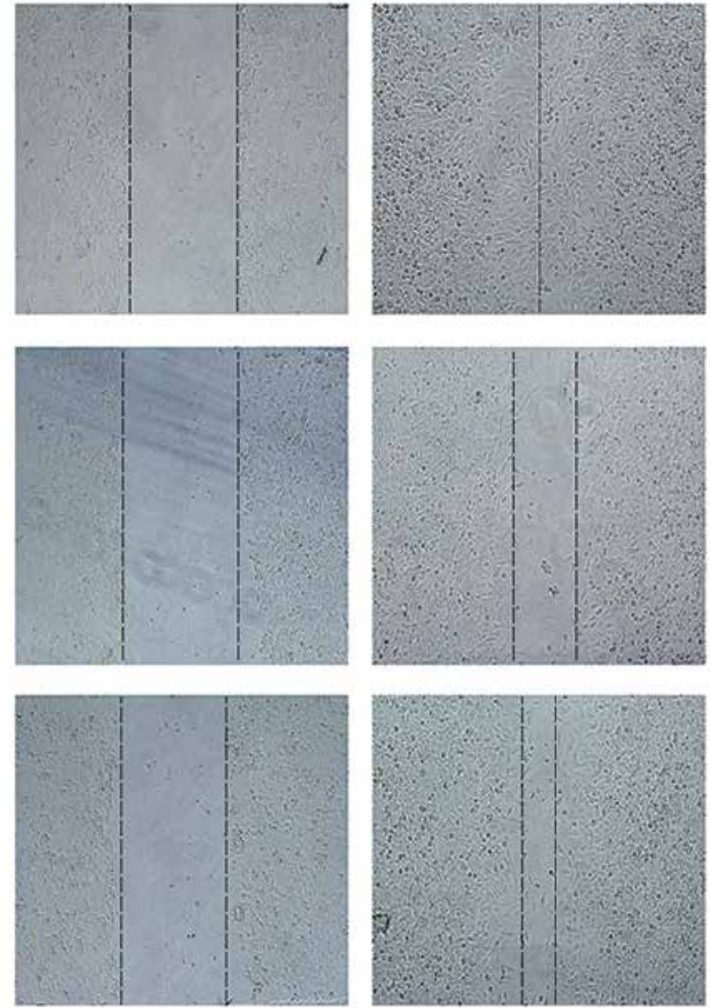
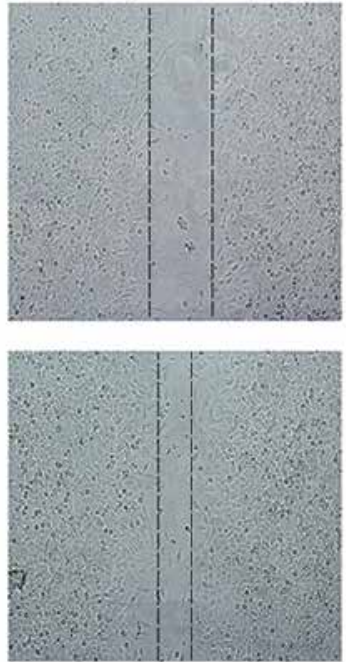

Figure 6. Cont. Overexpression of RCC2 restores the migration and invasion of EOC cells. Images of Figure 5 C (A), Figure $5 \mathrm{D}(\mathrm{B})$, and Figure $5 \mathrm{E}(\mathrm{C})$ are shown

In conclusion, our study revealed that miR-331-3p could significantly inhibit the proliferation, migration and invasion of EOC cells, and that RCC2 is the direct target of miR-331-3p in ovarian cancer. The findings from our study further clarify the role of miR-331-3p in ovarian carcinoma tumorigenesis, and demonstrate that the miR-331-3p /RCC2 signaling axis could be a novel tumor detection marker as well as a potential therapeutic target for ovarian carcinoma.

\section{Acknowledgments}

Gulimire Buranjiang and Reziya Kuerban are co-first authors this article.

\section{Conflict of interest}

The authors declare no conflict of interest.

\section{References}

1. Berek JS, Crum C, Friedlander M. Cancer of the ovary, fallopian tube, and peritoneum. Int J Gynaecol Obstet 2012; 119 Suppl 2: S118-29.

2. Siegel RL, Miller KD, Jemal A. Cancer statistics, 2015. CA Cancer J Clin 2015; 65: 5-29.

3. Dahiya N, Morin PJ. MicroRNAs in ovarian carcinomas. Endocr Relat Cancer 2010; 17: F77-89.

4. Feng Z, Wen H, Bi R, Yang W, Wu X. Prognostic impact of the time interval from primary surgery to intravenous chemotherapy in high grade serous ovarian cancer. Gynecol Oncol 2016; 141: 466-70.

5. Siegel RL, Miller KD, Jemal A. Cancer statistics, 2016. CA Cancer J Clin 2016; 66: 7-30.

6. Vargas-Hernandez VM, Moreno-Eutimio MA, Acosta-Altamirano G, Vargas-Aguilar VM. Management of recurrent epithelial ovarian cancer. Gland Surg 2014; 3: 198-202.

7. Iorio MV, Visone R, Di Leva G, et al. MicroRNA signatures in human ovarian cancer. Cancer Res 2007; 67: 8699-707.

8. Ambros V. The functions of animal microRNAs. Nature 2004; 431: 350-5.

9. Bartel DP. MicroRNAs: genomics, biogenesis, mechanism, and function. Cell 2004; 116: 281-97.

10. Deb B, Uddin A, Chakraborty S. miRNAs and ovarian cancer: an overview. J Cell Physiol 2018; 233: 3846-54.

11. Dong R, Liu X, Zhang Q, et al. miR-145 inhibits tumor growth and metastasis by targeting metadherin in highgrade serous ovarian carcinoma. Oncotarget 2014; 5: 10816-29.

12. Zhang H, Wang Q, Zhao Q, Di W. MiR-124 inhibits the migration and invasion of ovarian cancer cells by targeting SphK1. J Ovarian Res 2013; 6: 84.

13. Yeh YM, Chuang CM, Chao KC, Wang LH. MicroRNA-138 suppresses ovarian cancer cell invasion and metastasis by targeting SOX4 and HIF-1alpha. Int J Cancer 2013; 133: 867-78.

14. Zhang Y, Zhao FJ, Chen LL, et al. MiR-373 targeting of the Rab22a oncogene suppresses tumor invasion and metastasis in ovarian cancer. Oncotarget 2014; 5: 12291-303.

15. Guo F, Cogdell D, Hu L, et al. MiR-101 suppresses the epithelial-to-mesenchymal transition by targeting ZEB1 
and ZEB2 in ovarian carcinoma. Oncol Rep 2014; 31: 2021-8.

16. Shell S, Park SM, Radjabi AR, et al. Let-7 expression defines two differentiation stages of cancer. Proc Natl Acad Sci USA 2007; 104: 11400-5.

17. Shen J, Ambrosone CB, DiCioccio RA, Odunsi K, Lele SB, Zhao $\mathrm{H}$. A functional polymorphism in the miR-146a gene and age of familial breast/ovarian cancer diagnosis. Carcinogenesis 2008; 29: 1963-6.

18. Kinose Y, Sawada K, Nakamura K, Kimura T. The role of microRNAs in ovarian cancer. Biomed Res Int 2014 2014: 249393.

19. Pecot CV, Rupaimoole R, Yang D, et al. Tumour angiogenesis regulation by the miR-200 family. Nat Commun 2013; 4: 2427.

20. Cao Y, Chen J, Wang D, et al. Upregulated in hepatitis B virus-associated hepatocellular carcinoma cells, miR331-3p promotes proliferation of hepatocellular carcinoma cells by targeting ING5. Oncotarget 2015; 6 : 38093-106.

21. Chang RM, Yang H, Fang F, Xu JF, Yang LY. MicroRNA-331-3p promotes proliferation and metastasis of hepatocellular carcinoma by targeting PH domain and leucine-rich repeat protein phosphatase. Hepatology 2014; 60: 1251-63.

22. Fujii T, Shimada K, Asano A, et al. MicroRNA-331-3p suppresses cervical cancer cell proliferation and E6/E7 expression by targeting NRP2. Int J Mol Sci 2016; 17: 1351.

23. Guo X, Guo L, Ji J, et al. miRNA-331-3p directly targets E2F1 and induces growth arrest in human gastric cancer. Biochem Biophys Res Commun 2010; 398: 1-6.

24. Qi J, Zhao D, Cui M, Liu Q, Fang Q. MiR-331-3p is down-regulated in renal cell carcinoma and inhibits cell proliferation and invasion by targeting HER2 and NRP2. Int J Clin Exp Med 2017; 10: 16224-30

25. Zhao D, Sui $Y$, Zheng X. miR-331-3p inhibits proliferation and promotes apoptosis by targeting HER2 through the PI3K/Akt and ERK1/2 pathways in colorectal cancer. Oncol Rep 2016; 35: 1075-82.

26. Cannistra SA. Cancer of the ovary. N Engl J Med 2004; 351: 2519-29.

27. Bell DA. Origins and molecular pathology of ovarian cancer. Mod Pathol 2005; 18 Suppl 2: S19-32.

28. Pongsavee M. Effects of 744ins 20 - ter240 BRCA1 mutation on breast/ovarian carcinogenesis and the role of curcumin in telomerase inhibition. Arch Med Sci Civil Dis 2017; 2: 125-9.

29. Vaughan S, Coward JI, Bast RC Jr, et al. Rethinking ovarian cancer: recommendations for improving outcomes. Nat Rev Cancer 2011; 11: 719-25.

30. Epis MR, Giles KM, Beveridge DJ, et al. miR-331-3p and Aurora Kinase inhibitor II co-treatment suppresses prostate cancer tumorigenesis and progression. Oncotarget 2017; 8: 55116-34.

31. Pang B, Wu N, Guan RW, et al. Overexpression of RCC2 enhances cell motility and promotes tumor metastasis in lung adenocarcinoma by inducing epithelial-mesenchymal transition. Clin Cancer Res 2017; 23: 5598-610.

32. Cao Q, Li B, Wang X, Sun K, Guo Y. Therapeutic inhibition of CXC chemokine receptor 2 by SB225002 attenuates LPS-induced acute lung injury in mice. Arch Med Sci 2018; 14: 635-44

33. Matsuo M, Nakada C, Tsukamoto Y, et al. MiR-29c is downregulated in gastric carcinomas and regulates cell proliferation by targeting RCC2. Mol Cancer 2013; 12: 15.
34. Kang K, Huang YH, Li HP, Guo SM. Expression of UCA1 and MALAT1 long-chain non-coding RNAs in esophageal squamous cell carcinoma tissues is predictive of patient prognosis. Arch Med Sci 2018; 14: 752-9.

35. Mollinari C, Reynaud C, Martineau-Thuillier S, et al. The mammalian passenger is an RCC1 family member protein TD-60 with an essential role in prometaphase to metaphase progression. Dev Cell 2003; 5: 295-307.

36. Li JY, Liu CP, Shiao WC, et al. Inhibitory effect of PDGF$\mathrm{BB}$ and serum-stimulated responses in vascular smooth muscle cell proliferation by hinokitiol via up-regulation of p21 and p53. Arch Med Sci 2018; 14: 579-87.

37. Zhang Y, Wang Z, Zhang L, et al. Impact of connexin 43 coupling on survival and migration of multiple myeloma cells. Arch Med Sci 2017; 13: 1335-46. 